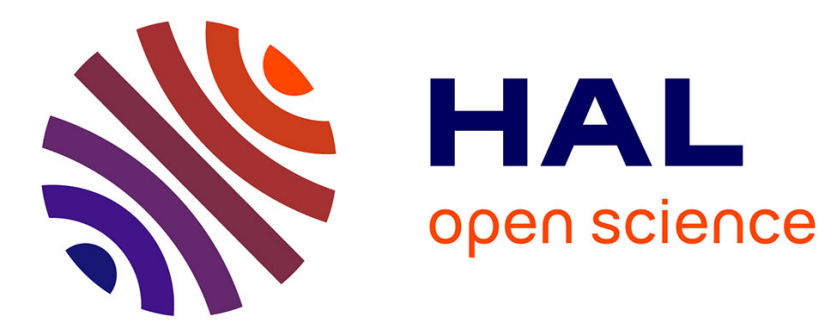

\title{
Multi-Antenna GNSS Receiver for Space Launcher
}

Jérémy Vezinet, Olivier Julien

\section{To cite this version:}

Jérémy Vezinet, Olivier Julien. Multi-Antenna GNSS Receiver for Space Launcher. ION GNSS+ 2017, 30th International Technical Meeting of The Satellite Division of the Institute of Navigation, Institute of navigation, Sep 2017, Portland, United States. pp. 1603-1612. hal-01705847

\section{HAL Id: hal-01705847 https://hal-enac.archives-ouvertes.fr/hal-01705847}

Submitted on 9 Feb 2018

HAL is a multi-disciplinary open access archive for the deposit and dissemination of scientific research documents, whether they are published or not. The documents may come from teaching and research institutions in France or abroad, or from public or private research centers.
L'archive ouverte pluridisciplinaire HAL, est destinée au dépôt et à la diffusion de documents scientifiques de niveau recherche, publiés ou non, émanant des établissements d'enseignement et de recherche français ou étrangers, des laboratoires publics ou privés. 


\title{
Multi-Antenna GNSS Receiver for Space Launcher
}

\author{
Jérémy Vezinet and Olivier Julien \\ SIGnal processing and NAVigation (SIGNAV) research group \\ TELECOM Laboratory \\ Ecole Nationale de l'Aviation Civile (ENAC)
}

\section{BIOGRAPHY (IES)}

Jérémy VEZINET graduated as an electronics engineer in 2010 and obtained his PhD in 2011 on multi-sensor hyrbidization from the ENAC (French National School for Civil Aviation) in Toulouse, France. Since 2011, he is a researcher and worked on multi-sensor hybridization research activities. He is now currently working on a multi-antenna GNSS receiver for space applications.

Olivier Julien is the head of the SIGnal processing and NAVigation (SIGNAV) research group of the TELECOM lab of ENAC (French Civil Aviation University), Toulouse, France. His research interests are GNSS receiver design, GNSS multipath and interference mitigation, and interoperability. He received his engineer degree in 2001 in digital communications from ENAC and his PhD in 2005 from the Department of Geomatics Engineering of the University of Calgary, Canada.

\begin{abstract}
This paper presents a multi-antenna GNSS solution for a launch vehicle able to provide position and velocity of the launcher continuously, including during the rotation and acceleration phases of flight. The originality of this work is the development of a multi-antenna, multi-constellation (GPS/GALILEO) and multi-frequency (L1/L5) GNSS positioning solution. The multiple antenna configuration is considered in order to keep tracking a sufficient number of GNSS signals during the whole flight. Under these improved visibility conditions, a navigation solution is proposed combining data received from all antennas to estimate the position and velocity of the launch vehicle. The proposed algorithm is an Extended Kalman Filter that uses code pseudoranges and doppler measurements. In the paper, several configurations and combinations are tested and compared.
\end{abstract}

\section{INTRODUCTION}

GNSS is today widely used in many navigation application: urban and terrestrial (for pedestrians, cyclists, automobiles or robots), maritime (for boats and ships), air navigation (for military and civil aircraft or UAVs) and spatial (for space or launch vehicles). Each of these domains can require a specific signal processing and navigation algorithm design based on the environment characteristics, the dynamic of the carrier or the requirement of the intended application.

For the particular case of space launchers, a significant variation in the attitude of the platform involves the use of multiple antennas located around the body in order to track a sufficient number of satellites during the flight. Several multi-antenna techniques have been addressed in the literature. Antenna diversity techniques are presented in [1] and [2] where a GNSS receiver uses combinations of signals from several antennas. Antenna diversity techniques offer a great improvement for radio link performance and uses redundancy of the received signal to increase the signal to noise ratio. Those techniques are usually employed to fight against signal fading. [3] and [4] also present antenna diversity techniques sounding rockets applications, vehicles that have high spinning movements, to keep tracking a sufficient number of satellite signals during the spinning motion. [8] presents an interesting approach by detailing a receiver that combines inputs from multiple antennas during signal tracking in order to keep a full sky visibility. The algorithm describes a fast-switching antenna selection process in order to ensure synchronization between the receiver and the incoming signal at every moment.

In addition to the desired multi-antenna architecture, the high dynamic motions of a launcher is a serious constraint for the design of a GNSS receiver. Indeed, acquisition and tracking of GNSS signals in presence of a non-null jerk is problematic since classic tracking loops have a limited capacity to follow frequency and phase variations induced by high dynamic motions. A launch 
vehicle is constantly submitted to high dynamic phases such as rotations or sudden accelerations (engines turn-on and turn-off) causing systematic losses of signal tracking.

One of the possible improvements for tracking loops used on GNSS receivers used in high dynamic vehicles can be the increase of the tracking loops' order. For example, [5] presents a controlled-root formulation for digital PLL with coefficients allowing to model a fourth order PLL. Another improvement for tracking loop designs under high dynamic constraints consists in using aided PLL as depicted in [6] and [7]. In that structure, the FLL-aided PLL design allows tracking accelerations steps happening during some phases of flight of a launch vehicle.

The solution proposed in the paper depicts a multi-antenna GNSS solution for a launch vehicle. The described solution offers a multi-antenna, dual-frequency and multi-constellation capability and assess positioning performances for a high dynamic range vehicle. The proposed algorithm is an extended Kalman filter using code pseudorange and Doppler measurements for estimation of the user's position and velocity. The paper is organized as follows:

- The first section describes the antenna and receiver configuration considered during our simulations. Number and positions of antennas are presented just as the considered tracking parameters of the receiver.

- The second section provides the description of the considered navigation filter.

- The third section presents the antenna switching strategy employed when some signals goes from one antenna to another. In such a case, the tracking channel parameters of one antenna can be used for aiding the acquisition of the same signal through another antenna.

- The fourth section details the algorithm performance assessment. Simulations in several configuration will be conducted:

- A first basic configuration aims at using all the antenna measurements in the EKF algorithms. Therefore, in this scenario no lever arm will be considered.

- The second configuration focuses on providing a navigation solution for each antenna. In this case the low number of visible satellites per antenna is an issue.

- The last configuration is the more complex one, it involves the antenna switching process integrates all measurement (one per satellite) in one extended Kalman filter and includes lever-arm compensation.

- The main conclusions of this paper, trade-off analysis and future work will be drawn in the last section.

\section{ANTENNA AND RECEIVER CONFIGURATION}

The system considered in this paper is composed of a GNSS receiver with three input antennas, mounted on a launch vehicle. Antennas are considered to be uniformly spaced around the cylindrical body of the launcher as illustrated in Figure 1. Three antennas are chosen in this configuration since their combined coverage should ensure that during every part of flight, all satellites in view are received whatever the attitude of the vehicle.

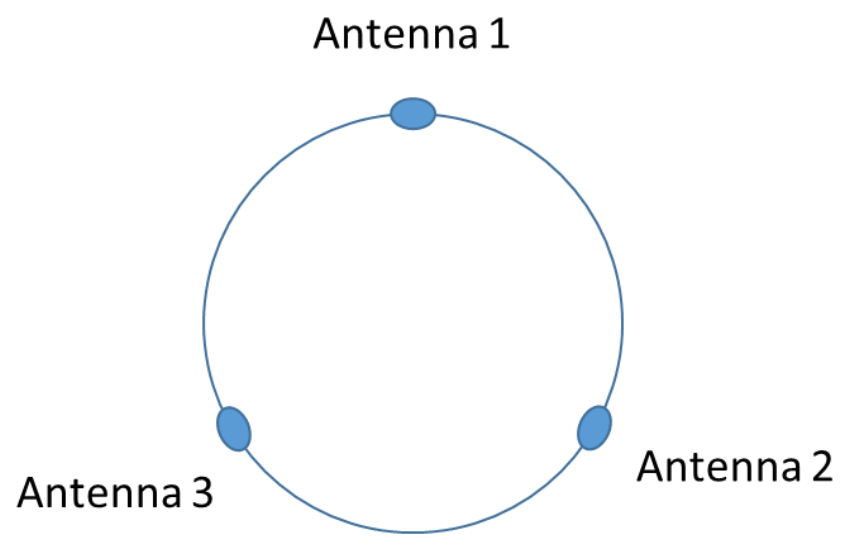

Figure 1. Spatial antenna distribution

The simulation presented in that paper use the GPS and Galileo constellations in the L1 and L5 frequency bands. Processing of the signals received by all antennas were based on the ENAC GNSS receiver simulator "GeneIQ". This receiver simulator works at the correlator output level. It thus employs very fine correlator output models including a large source of errors. For the purpose of this project, it has been adapted to be able to process signals from multiple antennas simultaneously and to take into account 
the attitude evolution of the platform. The position computation module has also been improved to take the multiple resulting measurements in consideration.

The navigation filter algorithm was executed at $100 \mathrm{~Hz}$. The GNSS receiver simulator allows testing several tracking architectures but for the presented simulations the configuration is as illustrated in the Table 1. The RF filter bandwidth is assumed to be of $24 \mathrm{MHz}$ (double-sided).

Table 1. Code and Carrier tracking parameters

\section{L1/L5/E1/E5 Code Tracking Parameters}

\begin{tabular}{|l|l|}
\hline DLL Order & 1 \\
\hline DLL noise bandwidth & $1 \mathrm{~Hz}$ \\
\hline DLL Integration time & $0.02 \mathrm{~s}$ \\
\hline Code Delay Discriminator & Early Minus Late Power (EMLP) \\
\hline GPS L1 chip spacing & $0.5 \mathrm{chip}$ \\
\hline GAL E1 chip spacing & $0.2 \mathrm{chip}$ \\
\hline GPS L5 chip spacing & $0.25 \mathrm{chip}$ \\
\hline GAL E5 chip spacing & $0.25 \mathrm{chip}$ \\
\hline L1/L5/E1/E5 Carrier Tracking Parameters & 3 \\
\hline PLL order & $20 \mathrm{~Hz}$ \\
\hline PLL noise bandwidth & $0.02 \mathrm{~s}$ \\
\hline PLL Integration time & Costas \\
\hline L1 C/A Carrier phase discriminator & Atan2 \\
\hline L5, E1C and E5A Carrier phase discriminator &
\end{tabular}

The choice of the tracking loop parameters (PLL order, bandwidth, and discriminator) has been set to these values for the current paper based on typical parameter values in the state-of-the-art. However, a study for optimizing the choice of these parameters is planned.

Note that the system configuration is based on the use of 3 receivers, each associated to one antenna. Moreover, it is assumed that these 3 receivers share a common oscillator in order to have a unique system time.

\section{NAVIGATION FILTER DESCRIPTION}

The proposed navigation filter is an extended Kalman filter (EKF). The filter uses code pseudoranges and Doppler measurements so as to estimate the vehicle's position and velocity. By definition, a Kalman filter is a recursive estimator of the internal state of a linear stochastic system. The EKF formulation allows dealing with nonlinear systems such as navigation systems.

\section{EKF State}

In the proposed EKF, the state is described by the following 8 state vector:

$$
X_{k}=\left[\begin{array}{llllllll}
x & \dot{x} & y & \dot{y} & z & \dot{z} & b_{C} & d_{C}
\end{array}\right]
$$

Where:

- $\quad(x, y, z)$ is the receiver's position vector in the ECEF system coordinates

- $\quad(\dot{x}, \dot{y}, \dot{z})$ is the receiver's velocity vector in the ECEF system coordinates

- $\quad b_{C}$ and $d_{C}$ are respectively the clock bias and clock drift of the receiver

Using the EKF, the estimation of the state vector $X_{k}$ is divided in two steps:

- The time prediction: This step consists in providing an estimate of the state vector $X_{k}$ (and of its covariance) based on its previous estimation $X_{k-1}$ and on the state transition model. The estimate of the state vector through the time prediction step is called the a-priori estimate and denoted $X_{k / k-1}$. 
- The measurement update: This step consists in updating (or correcting) the a priori estimate $X_{k / k-1}$ (and its covariance) by using the measurement vector $z_{k}$. The estimate of the state vector through the measurement update step is called the a-posteriori estimate and denoted $X_{k / k}$.

\section{State Transition Model}

The state transition model of the EKF filter in the continuous time domain can be expressed as follows:

$$
\dot{X}(t)=\varphi \cdot X(t)+w(t)
$$

Where:

- $\quad \dot{X}$ denotes the derivative of the state vector $X$

- $\quad w$ is the additive process noise vector assumed centered, white and Gaussian

- $\varphi$ is the continuous state transition matrix (Jacobian of the $f$ state transition function)

Then, in the discrete time domain, the transition state model becomes:

$$
X_{k}=\Phi \cdot X_{k-1}+w_{k}
$$

Where:

- $\quad X_{k}$ denotes the state vector forward projection from the $(k-1)^{\text {th }}$ to the $k^{\text {th }}$ time epoch

- $\Phi$ is the discrete state transition matrix and can be expressed as follows [9]:

$$
\Phi=\left[\begin{array}{cccc}
F & 0_{2 \times 2} & 0_{2 \times 2} & 0_{2 \times 2} \\
0_{2 \times 2} & F & 0_{2 \times 2} & 0_{2 \times 2} \\
0_{2 \times 2} & 0_{2 \times 2} & F & 0_{2 \times 2} \\
0_{2 \times 2} & 0_{2 \times 2} & 0_{2 \times 2} & F
\end{array}\right], F=\left[\begin{array}{cc}
1 & \Delta T \\
0 & 1
\end{array}\right]
$$

- $\Delta T$ denotes the time interval between two consecutive estimations, representing the measurement update time of the central filter

- $\quad w_{k}$ is the discrete process noise vector. It is modeled as a Gaussian noise vector with zero mean and discrete covariance matrix $Q_{k}$

The process noise comes from two sources namely [9], the user dynamic noise (constituted by the user's position and velocity terms) and the receiver's clock noise (local oscillator NCO noise).

The process noise is the first critical parameter affecting the performance of the filter. Therefore, an accurate tuning is required to fasten the EKF estimation convergence toward the true user state. Regarding the process noise covariance matrix, its discretetime form $Q_{k}$ is obtained from the continuous-time process noise matrix $Q$. The $Q$ matrix describes the uncertainty of the user's dynamic and is defined by:

$$
Q=E\left[w \cdot w^{T}\right]
$$

As depicted in [9], $Q$ covariance matrix contains:

- The user's dynamic sensitive terms $\left(\sigma_{\dot{x}}, \sigma_{\dot{y}}, \sigma_{\dot{z}}\right)$, mainly driven by the modeled user dynamic

- The receiver's oscillator noise terms including the oscillator's phase noise PSD, $\sigma_{b}$, and the oscillator's frequency noise $\mathrm{PSD}, \sigma_{d}$, which by themselves depend on the Allan variance parameters $h 0$ and $h-2$.

And can be expressed as follows:

$$
Q=\left[\begin{array}{cccc}
Q_{x} & 0 & 0 & 0 \\
0 & Q_{y} & 0 & 0 \\
0 & 0 & Q_{z} & 0 \\
0 & 0 & 0 & Q_{c l k}
\end{array}\right]
$$


With:

$$
\begin{aligned}
& \left.-Q_{x}=\left[\begin{array}{cc}
0 & 0 \\
0 & \sigma_{\dot{x}}^{2}
\end{array}\right] \text { (the same for } Q_{y} \text { and } Q_{z}\right) \\
& -\quad Q_{c l k}=\left[\begin{array}{cc}
\sigma_{b}^{2} & 0 \\
0 & \sigma_{d}^{2}
\end{array}\right]
\end{aligned}
$$

The discrete-time form of the process noise covariance sub-matrices $Q_{x}, Q_{y}$ and $Q_{z}$ can then be expressed:

$$
\begin{aligned}
Q_{x, k} & =\int_{t_{k-1}}^{t_{k-1}+\Delta T} \Phi_{x} \cdot Q_{x} \cdot \Phi_{x}^{T} \\
& =\int_{t_{k-1}}^{t_{k-1}+\Delta T}\left[\begin{array}{cc}
1 & \Delta T \\
0 & 1
\end{array}\right] \cdot\left[\begin{array}{cc}
0 & 0 \\
0 & \sigma_{\dot{x}}^{2}
\end{array}\right] \cdot\left[\begin{array}{cc}
1 & 0 \\
\Delta T & 1
\end{array}\right] \\
& =\left[\begin{array}{cc}
\Delta T^{3} / 3 & \Delta T^{2} / 2 \\
\Delta T^{2} / 2 & \Delta T
\end{array}\right]
\end{aligned}
$$

In an equivalent way, the receiver's clock process noise covariance discrete-time form can be expressed as follows:

$$
Q_{c l k, k}=\left[\begin{array}{cc}
\sigma_{b}^{2} \cdot \Delta T+\sigma_{d}^{2} \cdot \Delta T^{3} / 3 & \sigma_{d}^{2} \cdot \Delta T^{2} / 2 \\
\sigma_{d}^{2} \cdot \Delta T^{2} / 2 & \sigma_{d}^{2} \cdot \Delta T
\end{array}\right]
$$

\section{Measurement Model}

The measurement model describes the relation between the state of the system and the measurement vector. It is expressed as follows [9]:

$$
z_{k}=h\left(X_{k}\right)+v_{k}
$$

Where:

- $\quad h$ is the non-linear observation function that relates the measurement $z_{k}$ to the state vector $X_{k}$

- $\quad v_{k}$ is the measurement noise vector and is modelled as a zero-mean uncorrelated Gaussian noise process and independent to the process noise $w k$

- $\quad z_{k}$ is the measurement vector and is composed of the code pseudoranges and the Doppler measurements

Let $\rho_{i}$ and $\dot{\rho}_{i}$ respectively be the code pseudorange and Doppler measurements for a given satellite $i$. At each epoch $k$, these measurements are modeled as:

$$
\rho_{i}=h_{p}\left(X_{k}\right)+n_{p, k}
$$

Where:

$$
h_{p}\left(X_{k}\right)=r_{k}^{i}+c \cdot b_{C}
$$

$-\quad r_{k}^{i}=\sqrt{\left(x_{k}^{i}-x_{k}\right)^{2}+\left(y_{k}^{i}-y_{k}\right)^{2}+\left(z_{k}^{i}-z_{k}\right)^{2}}$ being the geometrical distance from the $i^{t h}$ satellite to the user at epoch $k$

- $\quad\left(x^{i}, y^{i}, z^{i}\right)$ being the coordinates of the $i^{\text {th }}$ satellite position in the ECEF frame

- $\quad n_{p}$ is the zero-mean Gaussian-distributed noise affecting the code pseudorange measurement

And

$$
\dot{\rho}_{i}=h_{d}\left(X_{k}\right)+n_{d, k}
$$

Where:

$$
h_{d}\left(X_{k}\right)=\left(\dot{x}_{k}^{i}-\dot{x}_{k}\right) \cdot a_{x}^{i}+\left(\dot{y}_{k}^{i}-\dot{y}_{k}\right) \cdot a_{y}^{i}+\left(\dot{z}_{k}^{i}-\dot{z}_{k}\right) \cdot a_{z}^{i}+c \cdot d_{C}
$$


- $\quad\left(a_{x}^{i}, a_{y}^{i}, a_{z}^{i}\right)$ denotes the line-of-sight unit vectors from the receiver to the $i^{\text {th }}$ satellite; $a_{x}^{i}=\frac{x_{k}^{i}-x_{k}}{r_{k}^{i}}, a_{y}^{i}=\frac{y_{k}^{i}-y_{k}}{r_{k}^{i}}, a_{z}^{i}=$ $\frac{z_{k}^{i}-z_{k}}{r_{k}^{i}}$

- $\quad\left(\dot{x}^{i}, \dot{y}^{i}, \dot{z}^{i}\right)$ being the velocity of the $i^{\text {th }}$ satellite in the ECEF frame

- $\quad n_{d}$ is the zero-mean Gaussian-distributed noise affecting the Doppler measurement

The measurement noise vector $v_{k}$ is modelled as a zero-mean uncorrelated Gaussian noise process and is independent to the process noise $w_{k}$. Its covariance matrix $R_{k}$ is defined by:

$$
R_{k}=E\left[v_{k} \cdot v_{k}^{T}\right]
$$

\section{ANTENNA SWITCHING PROCESS}

In order to deal with the high dynamic motion and the changes in attitude of a launch vehicle and ensure a sufficient number of received satellite signals, the multi-antenna structure has been considered in that study. Several solution adopting antenna diversity techniques have been presented in the literature ([3] and [4]). In our proposed solution, we choose not to combine signals from different antennas but to help tracking the signal of a new satellite seen from a given antenna with the information obtained when tracking the same signal from another antenna. That strategy ensures a reduction of the acquisition time in case of fast spinning motion when losing and re-acquiring a signal periodically. Figure 1 shows the number of satellites seen by each antenna during the flight.

In a similar way, Figure 3, illustrates the number of satellite signals that are considered as locked and that can be used in the navigation filter.

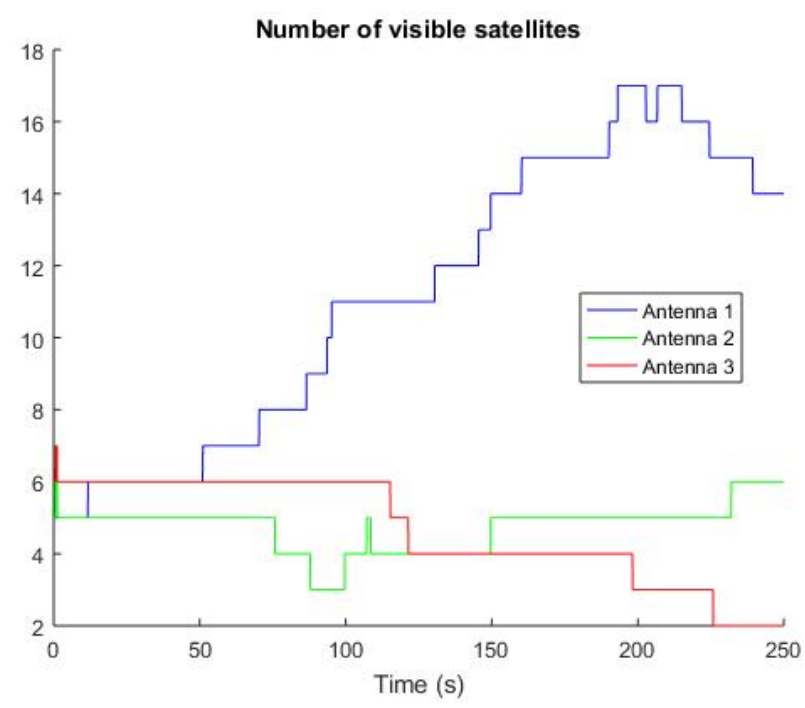

Figure 2. Number of visible satellites per antenna

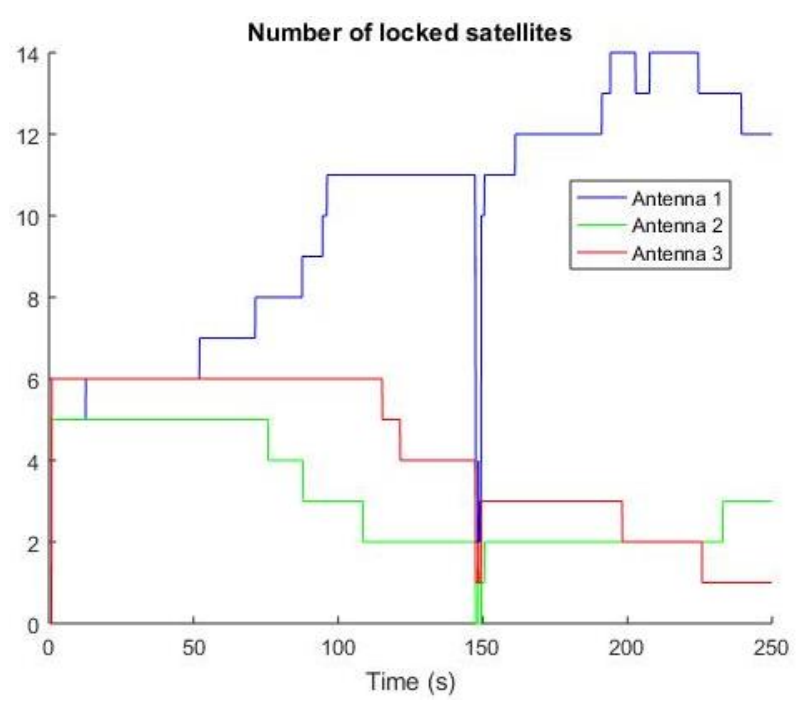

Figure 3. Number of locked satellites per antenna

The event at $t=150 \mathrm{~s}$ corresponds to a dropping phase during which the simulated trajectory is showing some singularities on the acceleration profile. The main consequence is the loss of lock of all signals. As the acquisition process was not studied in that paper, a perfect reacquisition is assumed a few seconds after that loss of lock.

Due to the proximity of the different antennas, it is very easy to initialize the DLL associated to a satellite newly seen by an antenna with the tracking parameters of the antenna already seeing that same antenna. However, this is not the case for the PLL. Indeed, the signals transmitted in the L1 and L5 bands by both GPS and Galileo constellations have wavelengths of around $19 \mathrm{~cm}$ and $25 \mathrm{~cm}$. These values are smaller than the distance between antennas so the carrier phase of arriving signal to different antennas may be different. Moreover, the rotation motion of the vehicle also impacts in different ways the Doppler associated to each antenna for a given satellite. The Doppler difference between antennas will depend upon the rotation motion of the vehicle, the 
relative position of each antenna and the location of the satellite. As a consequence, the only way to take properly into account this phase/Doppler difference between antennas would be to have access to an accurate estimation of the vehicle attitude and motion. Otherwise, the rotation of the vehicle would have to be slow. In this paper, it is reminded that each antenna is linked to a specific receiver (each receiver sharing the same oscillator). The proposed solution is thus as follows: when antenna 1 is receiving a new signal, which is currently being tracked by antenna 2, the tracking parameters associated to antenna 2 are used for initializing the antenna 1 tracking parameter. More precisely, phase and frequency estimated on antenna 2 are transmitted for the initialization of the carrier tracking loop of the antenna 1. That process can be optimized in the future by simulating fast rotation motion and assessing the switching performances.

Figure 4 and Figure 5 show estimated $\mathrm{C} / \mathrm{N}_{0}$ values for 2 satellites (PRN 8 and PRN37). For PRN 8, we see that antenna 3 is able to track the satellite until $t=90 \mathrm{~s}$. After that instant, antenna 1 is receiving the signal and antenna 2 is no longer tracking it. For PRN 37, there is a time interval during which antenna 1 and 3 are both tracking the satellite. During that phase, both antennas are able to track the signal and provide a measurement to the navigation filter. PLL associated to antenna 3 can provide to the PLL associated to antenna 1 the estimation of the frequency in order to start tracking the signal as soon as the satellite is in view.

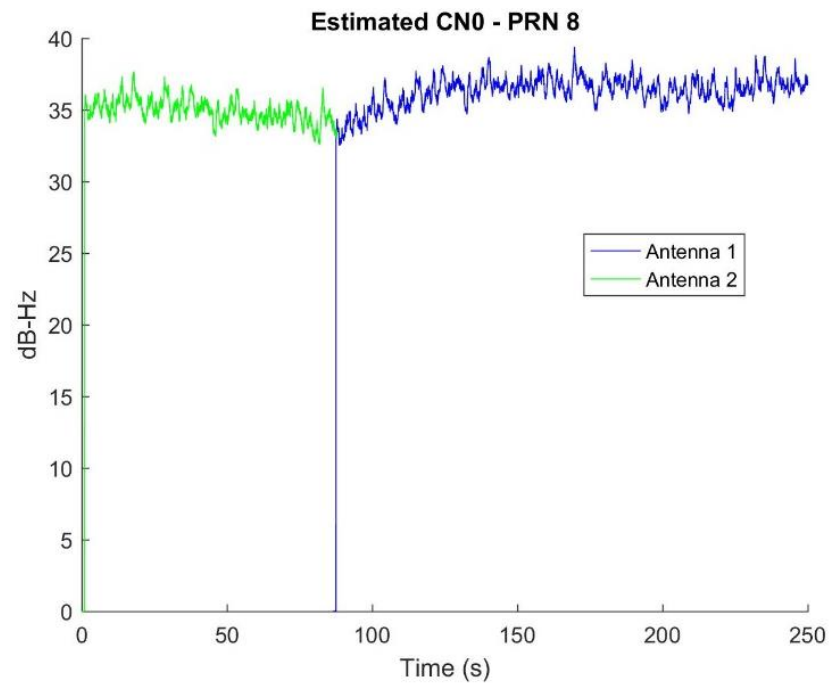

Figure 4. Estimated $\mathrm{C} / \mathrm{N}_{0}$ per antenna for PRN 8

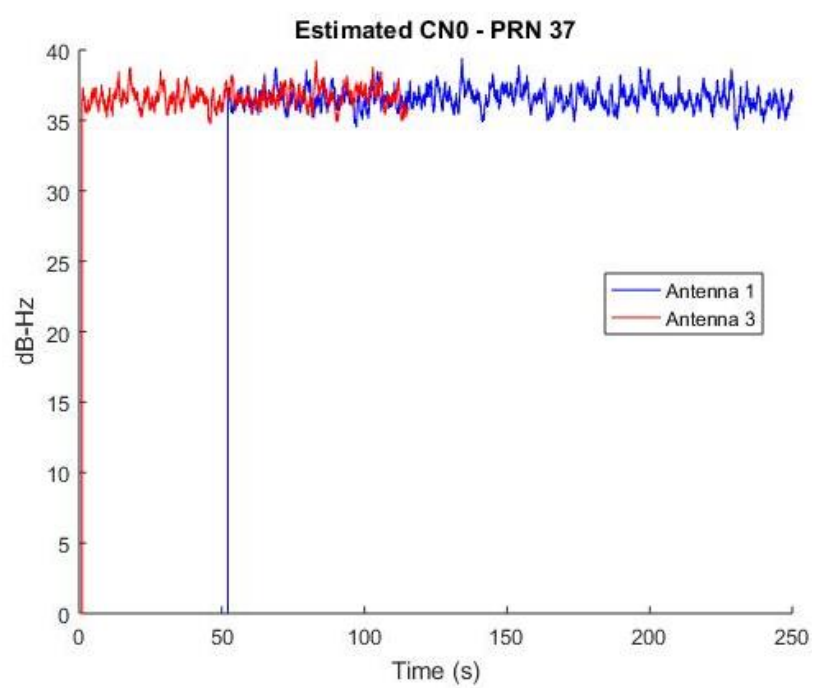

Figure 5. Estimated $\mathrm{C} / \mathrm{N}_{0}$ per antenna for $\mathrm{PRN} 37$

\section{PERFORMANCE ASSESSMENT}

In order to assess navigation performance of this solution and to propose improvements regarding the antenna, receiver or tracking loops configuration, several sets of simulations were performed. These simulations are assuming the presence of thermal noise only during the trajectory. As mentioned earlier, it is important to understand that the simulator used works at the correlator output level and that for the purpose of this project, it has been adapted to be able to process signals from multiple antennas simultaneously and to take into account the attitude evolution of the platform. The pseudorange measurements used by the positioning filter are Iono-Free measurements based on L1/L5 measurements. It is also assumed that if the receiver loses the L1 or the L5 signal, the corresponding IF measurement is unavailable (there is no reversion mode towards a single frequency measurement).

A first batch of independent Kalman filters, in which each of them is allocated to a specific antenna is used to assess performances of individual antennas solutions. These solutions deal with estimating position and velocity of each antennas without using the multi-antenna structure. However, even in a dual constellation configuration, some antennas can be masked by the vehicle body during some parts of the flight (see Figure 1) and the navigation solution is then degraded. Then the main drawback of these independent antenna solutions is the low number of measurement that can turn in a diverging navigation solution as soon as the antenna face a reduced part of the sky. Figure 6 and Figure 7 illustrate the KF solution estimation error per antenna. The dot lines correspond to the $3 \sigma$ estimated covariance by the Kalman filters. As soon as the number of locked satellites drops (for antennas 2 in green and antenna 3 in red), the estimated covariance as well as the estimation error increase and does not provide a reliable solution. In the simulated configuration, it reflects the fact that antenna 1 is facing up during most of the flight while antennas 2 
and 3 face down and thus have poor satellite visibility. As a comparison, Figure 8 and Figure 9 show a least square estimation of the position and velocity per antenna.
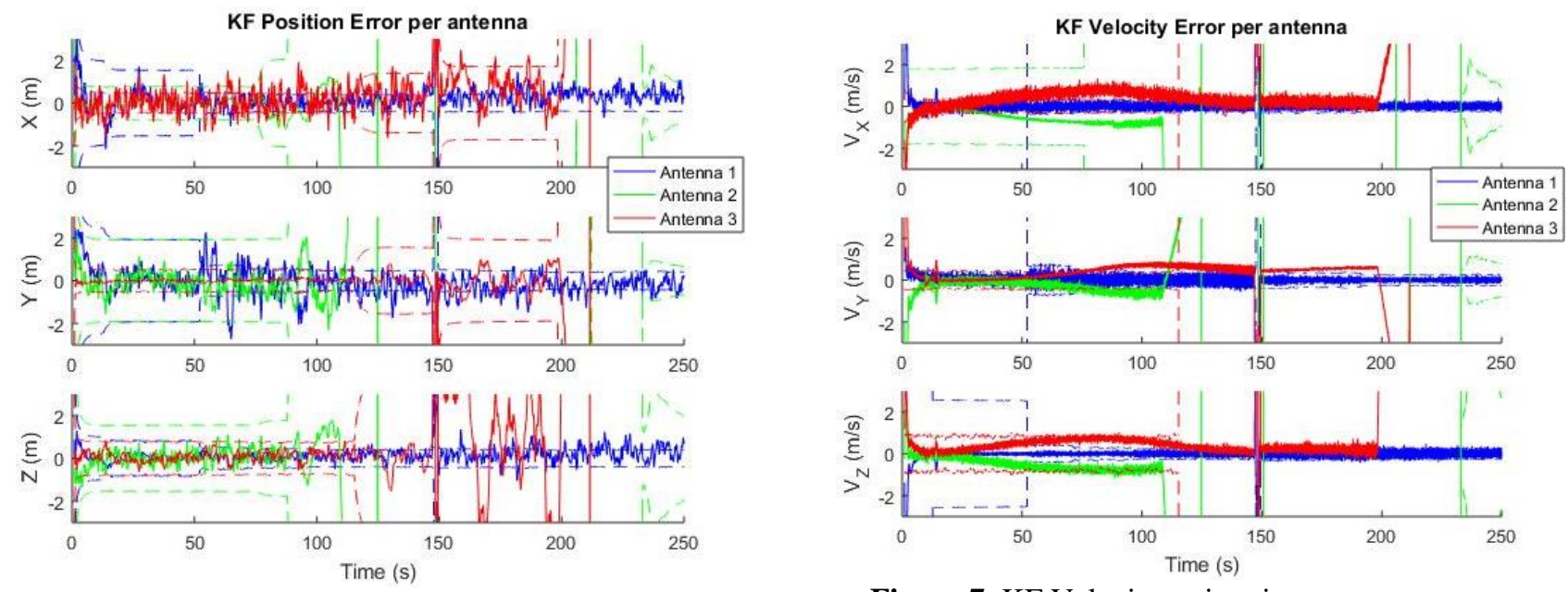

Figure 6. KF Position estimation error per antenna
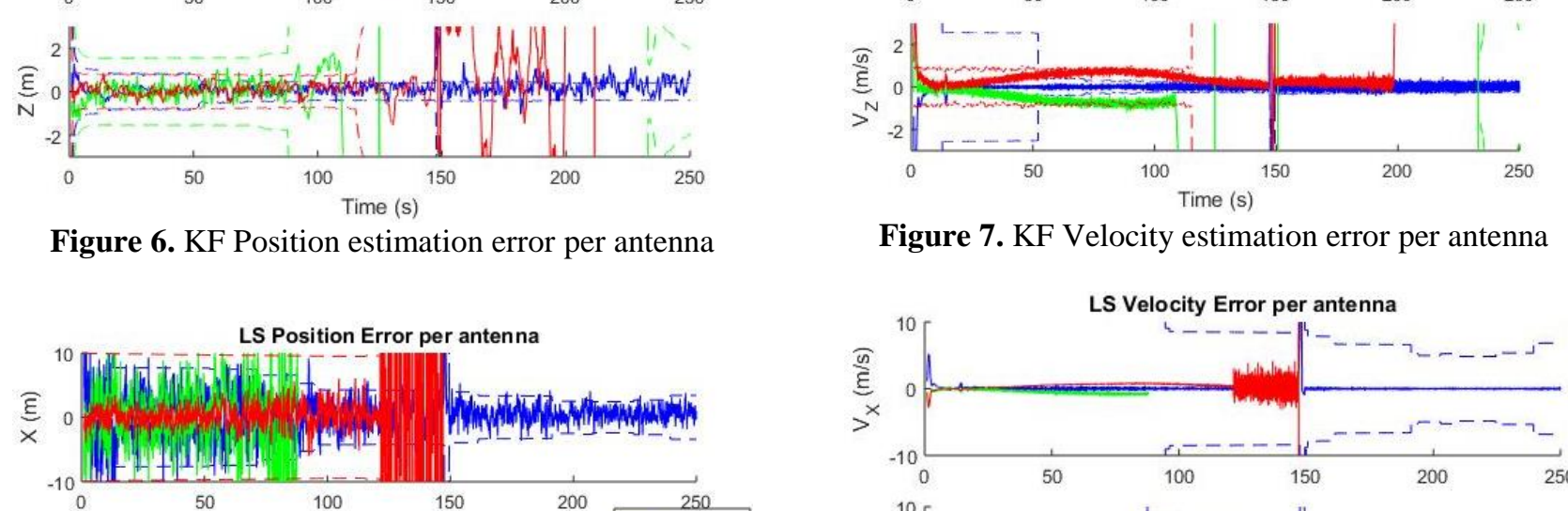

Figure 7. KF Velocity estimation error per antenna
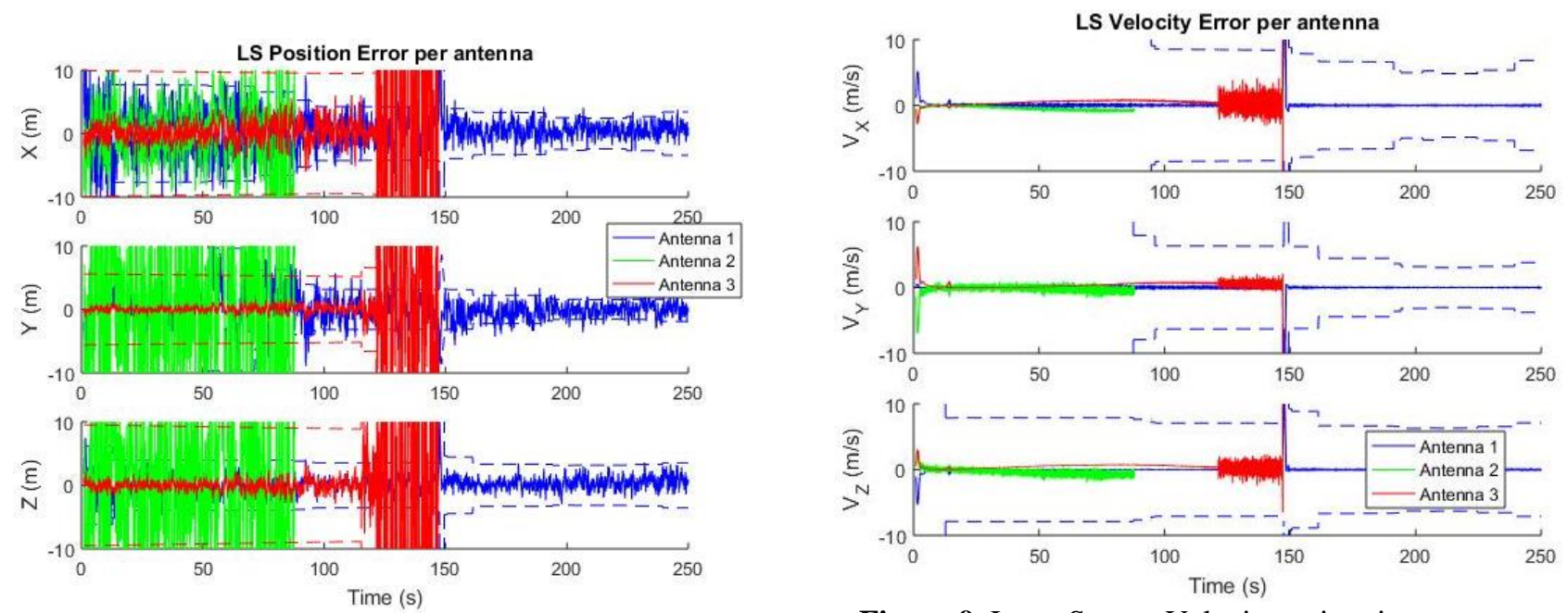

Figure 8. Least Square Position estimation error per antenna

Figure 9. Least Square Velocity estimation error per antenna

As mentioned before, the main drawback of these individual solutions is the decrease of the number of visible satellites per antenna during some parts of the flight. A first simple multi-antenna solution that do not solve this visibility issue but that may improve the navigation performance is to average the three individual antenna solutions. Even if the averaging will be impacted by the degraded solutions per antenna in some parts of the flight (especially when the number of locked satellite is low), it can provide a good first combined navigation solution. The position and velocity estimation errors appears in Figure 10 and Figure 11 in pink.

The next multi-antenna solution tested for this study is a solution that consists in using all measurement gathered from the three antennas for computing a global navigation solution. That global navigation focuses in estimating the position and velocity of the gravity center of the launch vehicle. All measurement associated to the three antennas are combined in a main EKF and in a first configuration, there is no compensation of the lever arm between the antenna and the vehicle's center of gravity. The lever arm is not compensated as it is assumed that there is no access with sufficient accuracy to the attitude of the vehicle. The position and velocity estimation errors are showed in Figure 10 and Figure 11 in black. The least square position and velocity estimation error are illustrated in Figure 12 and Figure 13.

The solution in which the lever arm are compensated is also simulated. In this case, the lever arm expressed in the vehicle frame is assumed to be well known. As presented in equation (1), position and velocity in the Kalman filter are expressed in the ECEF frame. The lever arm is then expressed in the ECEF frame so as to compute the innovation vector with a lever arm compensated 
predicted position. That frame rotation is done using an estimation of the vehicle's attitude. The estimation is assumed to be provided by an inertial aid during that simulation. The improvement in terms of performance of the estimated position and velocity is illustrated in Figure 10 and Figure 11 in blue. It can be used so as to justify the need of an inertial aid in the final solution.
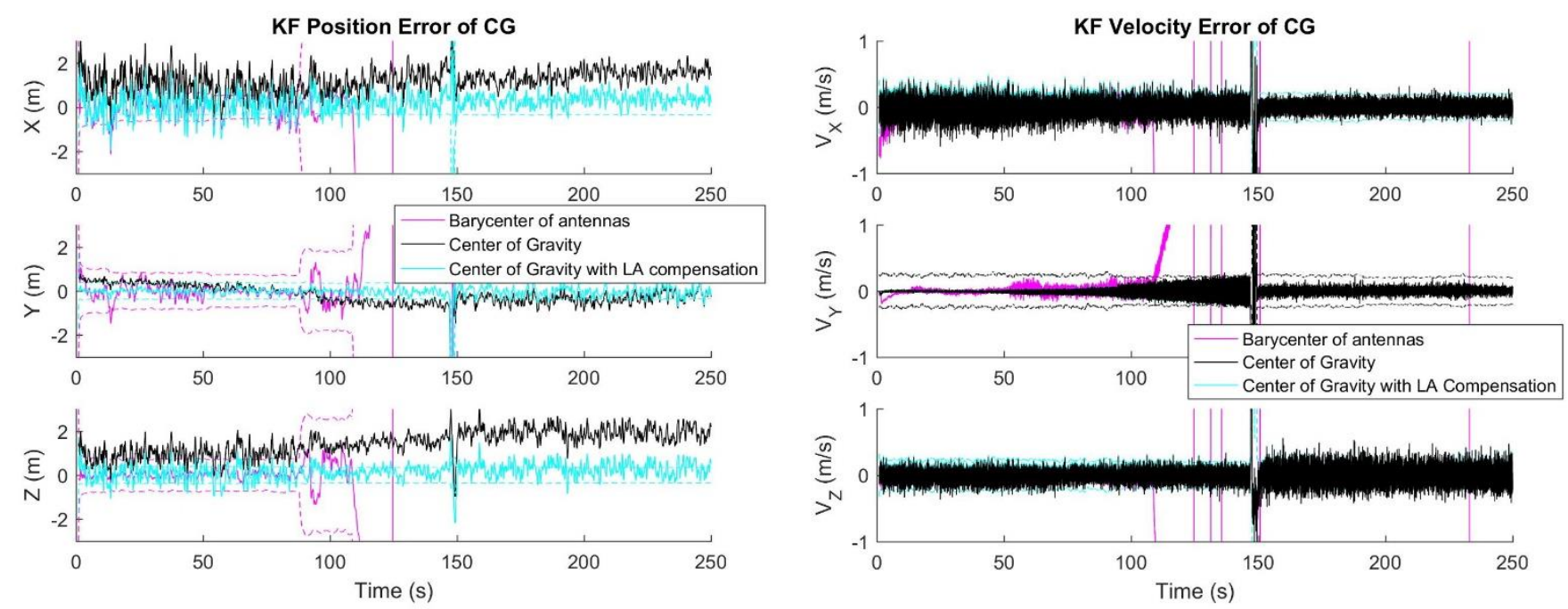

Figure 10. Global KF Position estimation error
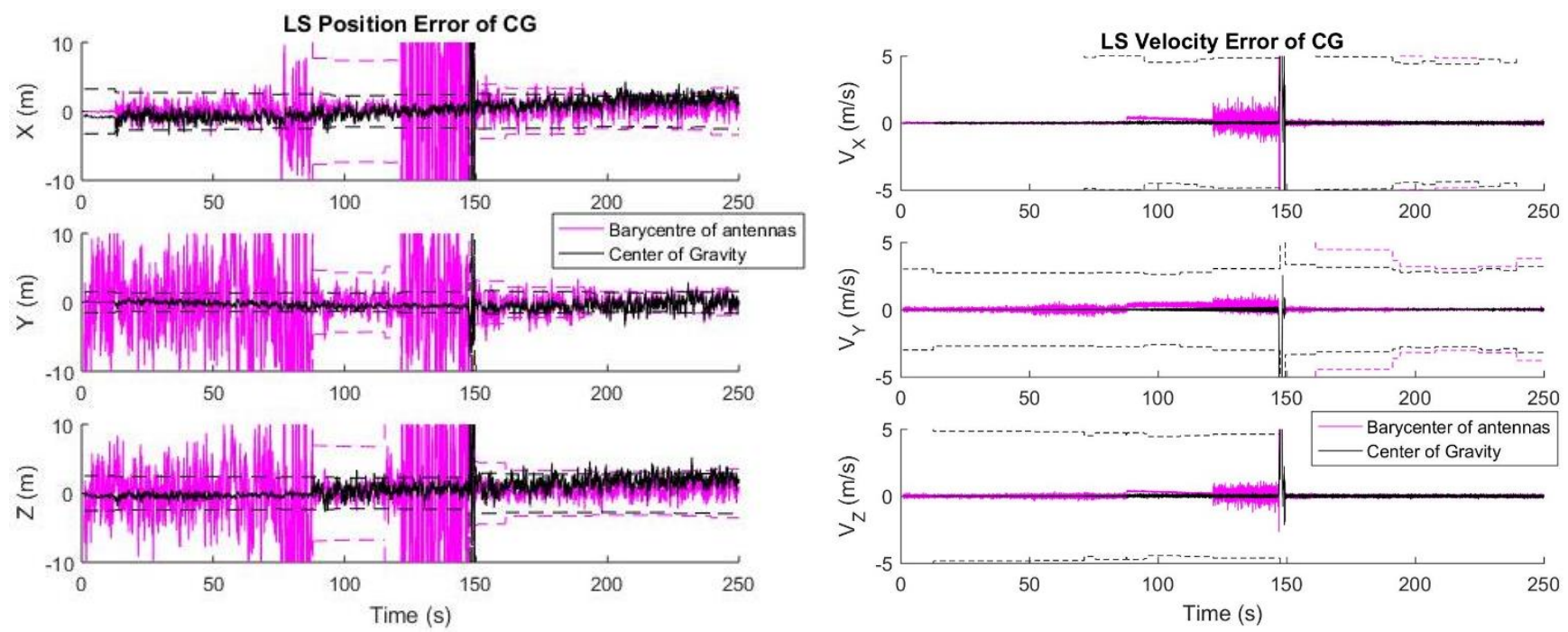

Figure 12. Global LS Position estimation error

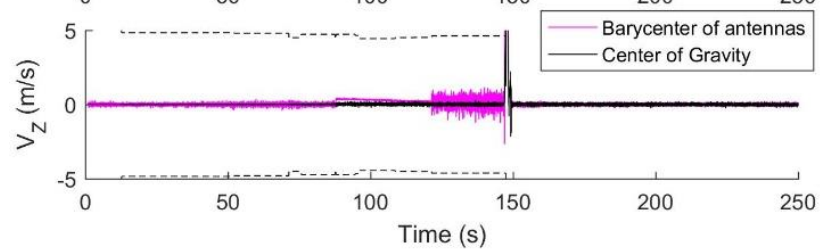

Figure 13. Global LS Velocity estimation error

The barycenter solution (in pink), as expected, drifts when one of the individual antenna solution drifts. This is due to the low number of satellite seen by antennas 2 and 3 after 100 seconds. That solution could be improved by detecting the reliable "per antenna" solution and discard the ones that are computed with a too low number of measurements.

The black plot shows the estimation errors for the solution that gather all measurements from all antennas but without lever arm compensation. A bias in the position estimation remains and could be an issue for large radius vehicles. In the current application a $2 \mathrm{~m}$ radius has been assumed.

The blue plot, which is identical to the black one but that uses lever arm compensation shows that there is no longer bias on the position estimation. In the two last configurations, the Kalman filter can integrate a sufficient number of measurement in order to provide a reliable position and velocity estimation. For the solution with lever arm compensation, the position on each axis reaches a sub-meter accuracy. 


\section{CONCLUSION AND FUTURE WORK}

The objective of this study is to propose a multi-antenna, multi-frequency and multi-constellation GNSS receivers. The solution is intended to be used for space launchers application and to deal with multiple constraints like the high dynamic of the vehicle or the masking of antennas by the vehicle. A position and velocity estimation algorithm using an extended Kalman Filter has been proposed integrating all measurement from the three antennas. A first set of results are provided based on simulations assessing the position and velocity estimation performance. An antenna switching strategy allowing to transmit estimated phase and frequency from an antenna to another has been used and will be improved in the future.

Those results offer a first analysis for refining the tracking loops architecture and parameters, the multi-antenna processing strategy and the navigation filter. Indeed, tracking parameters can be optimized so as to cope with the vehicle dynamics. Even if the increase of the carrier tracking loops bandwidth could have a significant effect on the noise at the measurement level and then on the navigation performances, it can help keeping lock of signals during high acceleration phases. Regarding the multiantenna strategy, even if a more complex process can help facing high rotation rates, the proposed way to initialize the PLL of an antenna with the estimated frequency from another antenna seems to be sufficient for the tested scenario. Finally, the navigation filter used in that solution (an 8-state EKF), could be improved by coupling the GNSS measurements to an IMU so as to estimate the fast changing attitude of the vehicle.

\section{ACKNOWLEDGMENTS}

This works has been done in the framework of a French national funding called "FUI REGLO". We would like to thank the leader of this project SYNTONY GNSS for its support.

\section{REFERENCES}

[1] S. W. Kim and Z. Wang, "Maximum Ratio Diversity Combining Receiver Using Single Radio Frequency Chain and Single Matched Filter," in Global Telecommunications Conference, 2007. GLOBECOM'07. IEEE, 2007.

[2] M. Akbari, M. R. Manesh, A. A. El-Saleh, and A. W. Reza, "Receiver Diversity Combining Using Evolutionary Algorithms in Rayleigh Fading Channel," Sci. World J., vol. 2014, pp. 1-11, 2014.

[3] A. Grillenberger and R. Rivas, "An Integrated Diversity Switching GPS Receiver for Sounding Rocket Missions," in International Global Navigation Satellite Systems Society, 2009.

[4] T. Ebinuma, H. Saito, K. Tanaka, and T. Miyoshi, "GPS Signal Tracking on Spinning Vehicles with Antenna Diversity Techniques," in 22nd International Technical Meeting of The Satellite Division of the Institute of Navigation, Savannah, Georgia, 2009.

[5] S. A. Stephens and J. B. Thomas, "Controlled-Root Formulation for Digital Phase-Locked Loops," IEEE Trans. Aerosp. Electron. Syst., vol. 31, no. 1, pp. 78-95, 1995.

[6] P. Roncagliolo, C. De Blasis, and C. Muravchik, "GPS Digital Tracking Loops Design for High Dynamic Launching Vehicles," in 2006 IEEE Ninth International Symposium on Spread Spectrum Techniques and Applications, 2006, pp. 4145 .

[7] P. A. Roncagliolo, J. G. García, and C. H. Muravchik, "Optimized Carrier Tracking Loop Design for Real-Time HighDynamics GNSS Receivers,” Int. J. Navig. Obs., vol. 2012, pp. 1-18, 2012.

[8] G. Puga, P. Roncagliolo, and J. Garcia, "Multi-antenna GNSS Receiver Tracking Algorithm for Vehicles With Unconstrained Three-dimensional Motion," presented at the 5th Argentine Symposium and Conference on Embedded Systems (SASE/CASE), 2014.

[9] E. Shytermeja, A. Garcia-Pena, and O. Julien, "Performance Evaluation of VDFLL Architecture for a Dual Constellation L1/E1 GNSS Receiver in Challenging Environments,” 2016. 